\title{
A Vector Equilibrium Problem for Muttalib-Borodin Biorthogonal Ensembles ${ }^{\star}$
}

Arno B.J. KUIJLAARS

Katholieke Universiteit Leuven, Department of Mathematics, Celestijnenlaan 200B box 2400, BE-3001 Leuven, Belgium

E-mail: arno.kuijlaars@kuleuven.be

URL: http://perswww.kuleuven.be/ u0017946/

Received April 12, 2016, in final form July 03, 2016; Published online July 05, 2016

http://dx.doi.org/10.3842/SIGMA.2016.065

\begin{abstract}
The Muttalib-Borodin biorthogonal ensemble is a joint density function for $n$ particles on the positive real line that depends on a parameter $\theta$. There is an equilibrium problem that describes the large $n$ behavior. We show that for rational values of $\theta$ there is an equivalent vector equilibrium problem.
\end{abstract}

Key words: biorthogonal ensembles; vector equilibrium problem; random matrix theory; logarithmic potential theory

2010 Mathematics Subject Classification: 31A05; 60B20

Dedicated to Percy Deift and Craig Tracy on the occasion of their 70th birthdays

\section{Introduction and statement of results}

\subsection{The Muttalib-Borodin ensemble}

The Muttalib-Borodin biorthogonal ensemble is the following probability density function for $n$ particles on the half line $[0, \infty)$

$$
\frac{1}{Z_{n}} \prod_{j<k}\left(x_{k}-x_{j}\right) \prod_{j<k}\left(x_{k}^{\theta}-x_{j}^{\theta}\right) \prod_{j=1}^{n} e^{-n V\left(x_{j}\right)}, \quad x_{j} \geq 0
$$

with $\theta>0$ and with an $n$-dependent weight function $w(x)=e^{-n V(x)}$ having enough decay at infinity. The model is named after Muttalib [17] who introduced it as a simplified model for disordered conductors in the metallic regime, and Borodin [4] who obtained profound mathematical results, in particular for Laguerre and Jacobi weights.

The model has attracted considerable attention in recent years. Random matrix models whose eigenvalues (or singular values) have the distribution $(1.1)$ were recently given in $[6,13]$. The model is also related to products of random matrices $[13,16]$.

In the large $n$ limit, the particles have an almost sure limiting measure $\mu^{*}$ which is the minimizer of

$$
\frac{1}{2} \iint \log \frac{1}{|x-y|} d \mu(x) d \mu(y)+\frac{1}{2} \iint \log \frac{1}{\left|x^{\theta}-y^{\theta}\right|} d \mu(x) d \mu(y)+\int V(x) d \mu(x)
$$

${ }^{\star}$ This paper is a contribution to the Special Issue on Asymptotics and Universality in Random Matrices, Random Growth Processes, Integrable Systems and Statistical Physics in honor of Percy Deift and Craig Tracy. The full collection is available at http://www.emis.de/journals/SIGMA/Deift-Tracy.html 
among all probability measures $\mu$ on $[0, \infty)$. This follows from large deviation results for (1.1) and related models that were studied in $[3,5,10]$. For $\theta=1$, the functional (1.2) reduces to the usual energy in the presence of an external field [20].

The minimizer for (1.2) was studied in detail by Claeys and Romano [7]. They found sufficient conditions for the minimizer to be supported on an interval $[0, a]$ for some $a>0$. Forrester and co-authors $[11,12]$ analyzed the equilibrium problem for (1.2) with special potentials, and found expressions for the minimizers as Fuss-Catalan and Raney distributions, see also [18].

It is the aim of this paper to show that for rational values of $\theta$, say $\theta=q / r$ with $q, r \in \mathbb{N}$, there is an equivalent minimization problem for a vector of $q+r-1$ measures. We expect that the vector equilibrium problem will be useful for subsequent asymptotic analysis. The special role of rational $\theta$ also appeared in the already mentioned work [7]. This paper gives finite term recurrence relations for the biorthogonal polynomials associated with (1.1), as well as a Christoffel-Darboux formula for the correlation kernel are given, but only for rational $\theta$.

In order to state our results we introduce the logarithmic energy

$$
I(\mu)=\iint \log \frac{1}{|x-y|} d \mu(x) d \mu(y)
$$

of a measure $\mu$ and the mutual energy

$$
I(\mu, \nu)=\iint \log \frac{1}{|x-y|} d \mu(x) d \nu(y)
$$

of two measures $\mu$ and $\nu$. Throughout we use for $j \in \mathbb{Z}$,

$$
\Delta_{j}= \begin{cases}{[0, \infty),} & \text { if } j \text { is even, } \\ (-\infty, 0], & \text { if } j \text { is odd }\end{cases}
$$

\subsection{Result for the case $\theta=1 / r$}

We first state the result for the case $\theta=1 / r$ with $r \in \mathbb{N}$.

Theorem 1.1. Let $V:[0, \infty) \rightarrow \mathbb{R}$ be continuous and

$$
\lim _{x \rightarrow \infty}\left(V(x)-\log \left(1+x^{2}\right)\right)=+\infty .
$$

Then there is a unique vector $\left(\mu_{0}^{*}, \mu_{1}^{*}, \ldots, \mu_{r-1}^{*}\right)$ of $r$ measures that minimizes the energy functional

$$
\sum_{j=0}^{r-1} I\left(\mu_{j}\right)-\sum_{j=0}^{r-2} I\left(\mu_{j}, \mu_{j+1}\right)+\int V d \mu_{0}
$$

among all vectors satisfying for every $j=0, \ldots, r-1$,

i) $\operatorname{supp}\left(\mu_{j}\right) \subset \Delta_{j}$,

ii) $\mu_{j}\left(\Delta_{j}\right)=1-\frac{j}{r}$, and

iii) $I\left(\mu_{j}\right)<+\infty$.

The measure $\mu_{0}^{*}$ has compact support, and it is the unique minimizer of the functional (1.2) with $\theta=1 / r$ among probability measures on $[0, \infty)$.

The minimization problem for the energy functional (1.6) is an example of a weakly admissible vector equilibrium problem in the sense of Hardy and Kuijlaars, see also below. The other measures $\mu_{1}^{*}, \ldots, \mu_{r-1}^{*}$ have full unbounded support, $\operatorname{supp}\left(\mu_{j}^{*}\right)=\Delta_{j}$ for $j=1, \ldots, r-1$. In fact $\mu_{j}^{*}=\frac{1}{2} \mathrm{Bal}\left(\mu_{j-1}^{*}+\mu_{j+1}^{*}, \Delta_{j}\right)$, where Bal denotes the balayage onto $\Delta_{j}$, see below as well. 


\subsection{Result for rational $\theta$}

For general rational $\theta=q / r$ with $q, r \in \mathbb{N}$, we first make the change of variables $x \mapsto x^{1 / q}$, $y \mapsto y^{1 / q}$ in the energy functional (1.2) to obtain

$$
\frac{1}{2} \iint \log \frac{1}{\left|x^{1 / q}-y^{1 / q}\right|} d \nu(x) d \nu(y)+\frac{1}{2} \iint \log \frac{1}{\left|x^{1 / r}-y^{1 / r}\right|} d \nu(x) d \nu(y)+\int \widehat{V}(x) d \nu(x),
$$

where $d \nu(x)=d \mu\left(x^{1 / q}\right)$, and $\widehat{V}(x)=V\left(x^{1 / q}\right)$. Note that $q$ and $r$ play a symmetric role in the energy functional (1.7).

Theorem 1.2. Let $\widehat{V}:[0, \infty) \rightarrow \mathbb{R}$ be continuous and

$$
\lim _{x \rightarrow \infty}\left(\widehat{V}(x)-\log \left(1+x^{2}\right)\right)=+\infty .
$$

Then there is a unique vector $\left(\nu_{-q+1}^{*}, \ldots, \nu_{-1}^{*}, \nu_{0}^{*}, \nu_{1}^{*}, \ldots, \nu_{r-1}^{*}\right)$ of $q+r-1$ measures that minimizes the energy functional

$$
\sum_{j=-q+1}^{r-1} I\left(\nu_{j}\right)-\sum_{j=-q+1}^{r-2} I\left(\nu_{j}, \nu_{j+1}\right)+\int \tilde{V} d \nu_{0}
$$

among all vectors satisfying for every $j=-q+1, \ldots, r-1$,

i) $\operatorname{supp}\left(\nu_{j}\right) \subset \Delta_{j}$,

ii) $\nu_{j}\left(\Delta_{j}\right)=m_{j}:= \begin{cases}1-\frac{j}{r} & \text { if } j \geq 0, \\ 1-\frac{|j|}{q} & \text { if } j \leq 0,\end{cases}$

iii) $I\left(\mu_{j}\right)<+\infty$.

The measure $\nu_{0}^{*}$ has compact support and it is the unique minimizer of the functional (1.7) among probability measures on $[0, \infty)$, and $d \mu_{0}^{*}(x)=d \nu_{0}^{*}\left(x^{q}\right)$ is the unique minimizer of $(1.2)$ with $\theta=q / r$.

As in Theorem 1.1 the other measures $\nu_{j}^{*}$ from Theorem 1.2 have full unbounded support: $\operatorname{supp}\left(\nu_{j}^{*}\right)=\Delta_{j}$ if $j \neq 0$.

The energy functional (1.9) with $\hat{V}=0$ and the normalizations $m_{j}$ as in condition ii) appeared in [9, Theorem 2.3] where it describes the limiting eigenvalue distribution of banded Toeplitz matrices. The supports $\Delta_{j}$ of the measures, however, are more general curves in that case.

Theorem 1.1 is the special case $q=1$ of Theorem 1.2 and it is enough to prove the latter theorem. However, for sake of exposition we chose to state Theorem 1.1 separately as well.

\section{Preliminaries}

\subsection{Vector equilibrium problems}

The unique existence of a minimizing vector of measures follows from the result of Hardy and Kuijlaars, which we recall here. The general setup of [14] involves the following ingredients.

(a) A sequence of closed subsets $\Delta_{1}, \ldots, \Delta_{d}$ of the complex plane, each with positive logarithmic capacity.

(b) For each $i=1, \ldots, d$, a lower semi-continuous function $V_{i}: \Delta_{i} \rightarrow \mathbb{R} \cup\{+\infty\}$ that is finite on a set of positive capacity. 
(c) A symmetric positive definite interaction matrix $C=\left(c_{i j}\right)$ of size $d \times d$.

(d) A sequence of positive numbers $m_{1}, \ldots, m_{d}$, such that

$$
\liminf _{|x| \rightarrow \infty, x \in \Delta_{i}}\left(V_{i}(x)-\left(\sum_{j=1}^{d} c_{i j} m_{j}\right) \log \left(1+|x|^{2}\right)\right)>-\infty
$$

for every $i=1, \ldots, d$ for which $\Delta_{i}$ is unbounded.

Associated with the above data is the energy functional

$$
J\left(\mu_{1}, \ldots, \mu_{d}\right)=\sum_{i, j=1}^{d} c_{i j} I\left(\mu_{i}, \mu_{j}\right)+\sum_{i=1}^{d} \int V_{i}(x) d \mu_{i}(x) .
$$

The problem is to minimize $J$ over $\prod_{j=1}^{d} \mathcal{M}_{m_{j}}\left(\Delta_{j}\right)$, or some subset of it. Here we use $\mathcal{M}_{m}(\Delta)$ to denote the set of positive Borel measures on $\Delta$ of total mass $m>0$. Such a minimization problem is called a weakly admissible vector equilibrium problem.

The functional (2.2) is not well-defined on all of $\prod_{j=1}^{d} \mathcal{M}_{m_{j}}\left(\Delta_{j}\right)$, since there is a problem with measures having overlapping supports, and with measures having unbounded supports. The problem with overlapping supports was solved by Beckermann et al. [2]. To handle the situation with unbounded supports, a regularization of $J$ is introduced in [14, Theorem 2.6] that comes from mapping the measures to the Riemann sphere and redefining $J$ accordingly. This procedure involves a modification of the mutual energy (1.4) and the corresponding logarithmic energy (1.3) to

$$
\tilde{I}(\mu, \nu)=\iint \log \left(\frac{\sqrt{1+|x|^{2}} \sqrt{1+|y|^{2}}}{|x-y|}\right) d \mu(x) d \nu(y),
$$

and $\tilde{I}(\mu)=\tilde{I}(\mu, \mu)$, respectively.

We may call $\tilde{I}(\mu)$ and $\tilde{I}(\mu, \nu)$ the spherical (mutual) energy. Then

$$
\tilde{J}\left(\mu_{1}, \ldots, \mu_{d}\right)= \begin{cases}\sum_{i, j=1}^{d} c_{i j} \tilde{I}\left(\mu_{i}, \mu_{j}\right)+\sum_{i=1}^{d} \int \tilde{V}_{i}(x) d \mu_{i}(x), & \text { if all } \tilde{I}\left(\mu_{i}\right)<+\infty, \\ +\infty, & \text { otherwise },\end{cases}
$$

where

$$
\tilde{V}_{i}(x)=V_{i}(x)-\left(\sum_{j=1}^{d} c_{i j} m_{j}\right) \log \left(1+|x|^{2}\right)
$$

is an extension of (2.2) since for vectors of measures with $I\left(\mu_{i}\right)<+\infty$ for all $i$, we have that $\tilde{J}\left(\mu_{1}, \ldots, \mu_{d}\right)=J\left(\mu_{1}, \ldots, \mu_{d}\right)$. Note that $\tilde{V}_{i}$ is bounded away from $-\infty$ on $\Delta_{i}$ because of the lower-semicontinuity of $V_{i}$ and the growth condition (2.1) at infinity.

The functional (2.3) is thus defined on $\prod_{j=1}^{d} \mathcal{M}_{m_{j}}\left(\Delta_{j}\right)$ with values in $\mathbb{R} \cup\{+\infty\}$ only. In addition, $\tilde{J}$ has compact sublevel sets $\tilde{J}^{-1}((-\infty, \alpha])$ for any real $\alpha$ (which implies that it is lower semi-continuous), and it is strictly convex on the set where it is finite. As a consequence, there is a unique minimizer of $\tilde{J}$ on $\prod_{j=1}^{d} \mathcal{M}_{m_{j}}\left(\Delta_{j}\right)$, see [14, Corollary 2.7]. 


\subsection{Variational conditions}

We use $U^{\nu}(z)=\int \log \frac{1}{|z-y|} d \nu(y), z \in \mathbb{C}$, to denote the logarithmic potential of a measure $\nu$ and

$$
\tilde{U}^{\nu}(z)=\int \log \left(\frac{\sqrt{1+|z|^{2}} \sqrt{1+|y|^{2}}}{|z-y|}\right) d \nu(y)
$$

to denote the spherical potential.

The variational conditions for the vector equilibrium problem were not discussed in [14]. The following result is standard for the case $d=1$, and its extension to $d \geq 2$ is not difficult.

Lemma 2.1. Let $\vec{\mu}^{*}=\left(\mu_{1}^{*}, \ldots, \mu_{d}^{*}\right)$ be a vector of measures in $\prod_{j=1}^{d} \mathcal{M}_{m_{j}}\left(\Delta_{j}\right)$ with $\tilde{I}\left(\mu_{i}^{*}\right)<+\infty$ for all $i$. Suppose that for some constants $\ell_{i}$,

$$
2 \sum_{j=1}^{d} c_{i j} \tilde{U}^{\mu_{j}^{*}}+\tilde{V}_{i} \begin{cases}=\ell_{i} & \text { on } \operatorname{supp}\left(\mu_{i}^{*}\right), \\ \geq \ell_{i} & \text { on } \Delta_{i},\end{cases}
$$

for every $i=1, \ldots, d$. Then $\vec{\mu}$ is the minimizer of $\tilde{J}$ in $\prod_{j=1}^{d} \mathcal{M}_{m_{j}}\left(\Delta_{j}\right)$.

Proof. The functional $\tilde{J}$ is strictly convex on the set where it is finite. The strict convexity is due to the quadratic part $\tilde{J}_{0}(\vec{\mu})=\sum_{i, j=1}^{d} c_{i j} \tilde{I}\left(\mu_{i}, \mu_{j}\right)$ in the functional $\tilde{J}$ and it comes down to

$$
\tilde{J}_{0}(\vec{\mu})+\tilde{J}_{0}(\vec{\nu}) \geq 2 \sum_{i, j=1}^{d} c_{i j} I\left(\mu_{i}, \nu_{j}\right),
$$

whenever $\vec{\mu}, \vec{\nu} \in \prod_{j=1}^{d} \mathcal{M}_{m_{j}}\left(\Delta_{j}\right)$, with strict inequality if $\vec{\mu} \neq \vec{\nu}$ and both $\tilde{J}(\vec{\mu})<+\infty, \tilde{J}(\vec{\nu})<+\infty$.

Let $\vec{\mu}^{*}$ be as in the lemma. Then for any $\vec{\mu} \in \prod_{j=1}^{d} \mathcal{M}_{m_{j}}\left(\Delta_{j}\right)$, we find by integrating (2.5) with respect to $\mu_{i}$,

$$
\int\left(2 \sum_{j=1}^{d} c_{i j} \tilde{U}^{\mu_{j}^{*}}+\tilde{V}_{i}\right) d \mu_{i} \geq \ell_{i} m_{i},
$$

and so by summing over $i$,

$$
2 \sum_{i, j=1}^{d} c_{i j} \tilde{I}\left(\mu_{i}, \mu_{j}^{*}\right)+\sum_{i=1}^{d} \int \tilde{V}_{i} d \mu_{i} \geq \sum_{i=1}^{d} \ell_{i} m_{i} .
$$

If we integrate $(2.5)$ with respect to $\mu_{i}^{*}$ and sum over $i$, we find an equality

$$
2 \sum_{i, j=1}^{d} c_{i j} \tilde{I}\left(\mu_{i}^{*}, \mu_{j}^{*}\right)+\sum_{i=1}^{d} \int \tilde{V}_{i} d \mu_{i}^{*}=\sum_{i=1}^{d} \ell_{i} m_{i},
$$

which means

$$
\sum_{i=1}^{d} \ell_{i} m_{i}=\tilde{J}\left(\vec{\mu}^{*}\right)+\tilde{J}_{0}\left(\vec{\mu}^{*}\right)
$$

and in particular $\tilde{J}\left(\vec{\mu}^{*}\right)<+\infty$. 
Now we use (2.6) with $\vec{\nu}=\vec{\mu}^{*}$, and combine it with (2.7) and (2.8) to find

$$
\begin{aligned}
\tilde{J}(\vec{\mu}) & =\tilde{J}_{0}(\vec{\mu})+\sum_{i=1}^{d} \int \tilde{V}_{i} d \mu_{i} \geq 2 \int \sum_{i, j=1}^{d} c_{i j} I\left(\mu_{i}, \mu_{j}^{*}\right)-\tilde{J}_{0}\left(\vec{\mu}^{*}\right)+\sum_{i=1}^{d} \int \tilde{V}_{i} d \mu_{i} \\
& \geq \sum_{i=1}^{d} \ell_{i} m_{i}-\tilde{J}_{0}\left(\vec{\mu}^{*}\right)=\tilde{J}\left(\vec{\mu}^{*}\right) .
\end{aligned}
$$

Thus $\vec{\mu}^{*}$ is indeed the minimizer of $\tilde{J}$.

\subsection{Nikishin interaction and balayage}

In the present paper we are dealing with the interaction matrix $C=\left(c_{i j}\right)$ where

$$
c_{i j}= \begin{cases}1, & \text { if } i=j \\ -\frac{1}{2}, & \text { if }|i-j|=1 \\ 0, & \text { otherwise }\end{cases}
$$

which is indeed a positive definite matrix, and sets $\Delta_{j}$ that alternate between the positive and negative real axis as in (1.5). The interaction matrix (2.9) is characteristic for Nikishin systems in the theory of Hermite-Padé approximation [19]. See also [1, 15] for surveys on the connections with Hermite-Padé approximation and random matrix theory.

In Theorem 1.2 we have $d=q+r-1$ and we use indices $i=-q+1, \ldots, r-1$, instead of $i=1, \ldots, d$. The total masses $m_{j}$ are as in condition ii) of Theorem 1.2. The external fields are $V_{0}=\widehat{V}$ and $V_{i} \equiv 0$ for $i \neq 0$. Then it is easy to see that the conditions (a), (b), (c), and (d) stated in Section 2.1 are satisfied. In fact we have (2.1), since

$$
\sum_{j=-q+1}^{r-1} c_{i j} m_{j}=-\frac{1}{2} m_{i-1}+m_{i}-\frac{1}{2} m_{i+1}= \begin{cases}0, & \text { for } i \neq 0 \\ \frac{q^{-1}+r^{-1}}{2}, & \text { for } i=0\end{cases}
$$

and then (2.1) is satisfied because of (1.8).

Hence there exists a unique minimizing vector of measures $\left(\nu_{-q+1}^{*}, \ldots, \nu_{r-1}^{*}\right)$ for the energy functional $\tilde{J}$ with

$$
\tilde{V}(x)=\widehat{V}(x)-\frac{q^{-1}+r^{-1}}{2} \log \left(1+|x|^{2}\right),
$$

see (2.4). We have to show that in addition $I\left(\nu_{i}^{*}\right)<+\infty$ for all $i$, and then we can conclude that it is also a minimizing vector for $J$.

The variational conditions (2.5) from Lemma 2.1 are in this case

$$
2 \tilde{U}^{\nu_{0}^{*}}-\tilde{U}^{\nu_{-1}^{*}}-\tilde{U}^{\nu_{1}^{*}}+\tilde{V} \begin{cases}=\ell_{0} & \text { on } \operatorname{supp}\left(\nu_{0}^{*}\right), \\ \geq \ell_{0} & \text { on }[0, \infty)\end{cases}
$$

and for $j \in\{-q+1, \ldots, r-1\} \backslash\{0\}$,

$$
2 \tilde{U}^{\nu_{j}^{*}}-\tilde{U}^{\nu_{j-1}^{*}}-\tilde{U}^{\nu_{j+1}^{*}} \begin{cases}=\ell_{j} & \text { on } \operatorname{supp}\left(\nu_{j}^{*}\right), \\ \geq \ell_{j} & \text { on } \Delta_{j} .\end{cases}
$$

Here we set $\nu_{-q}^{*}=\nu_{r}^{*}=0$ so that (2.10), (2.11) also hold for $j=-q+1$ and $j=r-1$.

Note that $2 \nu_{j}^{*}$ and $\nu_{j-1}^{*}+\nu_{j+1}^{*}$ have the same total masses if $j \neq 0$. If (2.11) holds then $2 \nu_{j}^{*}$ is the balayage measure of $\nu_{j-1}^{*}+\nu_{j+1}^{*}$ onto $\Delta_{j}$. The balayage measure has full support $\Delta_{j}$, and 
equality holds in (2.11) everywhere on $\Delta_{j}$. In addition, the constant $\ell_{j}$ is zero. So for $j \neq 0$ the variational condition is

$$
2 \tilde{U}^{\nu_{j}^{*}}-\tilde{U}^{\nu_{j-1}^{*}}-\tilde{U}^{\nu_{j+1}^{*}}=0 \quad \text { on } \Delta_{j} .
$$

If $x \mapsto \log \left(1+|x|^{2}\right)$ is integrable with respect to all measures, then (2.10) reduces to

$$
2 U^{\nu_{0}^{*}}-U^{\nu_{-1}^{*}}-U^{\nu_{1}^{*}}+\widehat{V} \begin{cases}=\ell & \text { on } \operatorname{supp}\left(\nu_{0}^{*}\right), \\ \geq \ell & \text { on }[0, \infty),\end{cases}
$$

for some constant $\ell$, while (2.12) reduces to

$$
2 U^{\nu_{j}^{*}}=U^{\nu_{j-1}^{*}}+U^{\nu_{j+1}^{*}} \quad \text { on } \quad \Delta_{j},
$$

which is the more common form for the balayage in logarithmic potential theory, see [20] where the discussion however is restricted to measures with compact support in $\mathbb{C}$.

Our strategy to prove Theorem 1.2 will be to establish the existence of a vector of measures $\vec{\nu}^{*}=\left(\nu_{-q+1}^{*}, \ldots, \nu_{r-1}^{*}\right)$ with $\operatorname{supp}\left(\nu_{j}^{*}\right) \subset \Delta_{j}, \nu_{j}^{*}\left(\Delta_{j}\right)=m_{j}$, such that the conditions $(2.14)$ and (2.13) are satisfied. The measure $\nu_{0}^{*}$ will have compact support, and all other measures have full support. The density of $\nu_{j}^{*}$ will behave like

$$
\frac{d \nu_{j}^{*}(x)}{d x}=\left\{\begin{array}{ll}
O\left(|x|^{-1-1 / q}\right) & \text { for } j \geq 1, \\
O\left(|x|^{-1-1 / r}\right) & \text { for } j \leq-1,
\end{array} \quad \text { as }|x| \rightarrow \infty .\right.
$$

\section{Proof of Theorem 1.2}

\subsection{An auxiliary result}

The following is our main auxiliary result.

Proposition 3.1. Let $r \geq 2$ be an integer and let $a>0$. Then there is a unique vector $\left(\mu_{1}^{*}, \ldots, \mu_{r-1}^{*}\right)$ of measures that minimizes the energy functional

$$
\sum_{j=1}^{r-1} I\left(\mu_{j}\right)-\sum_{j=1}^{r-2} I\left(\mu_{j}, \mu_{j+1}\right)+\int \log |x-a| d \mu_{1}(x)
$$

among all vectors of measures satisfying for every $j=1, \ldots, r-1$,

i) $\operatorname{supp}\left(\mu_{j}\right) \subset \Delta_{j}$,

ii) $\mu_{j}\left(\Delta_{j}\right)=1-\frac{j}{r}$, and

iii) $I\left(\mu_{j}\right)<+\infty$.

Moreover,

$$
\sum_{j=1}^{r-1} I\left(\mu_{j}^{*}\right)-\sum_{j=1}^{r-2} I\left(\mu_{j}^{*}, \mu_{j+1}^{*}\right)=-\frac{1}{2} \int \log |x-a| d \mu_{1}^{*}(x)
$$

and

$$
U^{\mu_{1}^{*}}(z)=\log \left|\frac{z^{1 / r}-a^{1 / r}}{z-a}\right|, \quad z \in \mathbb{C} .
$$


Proof. The minimization of (3.1) under the conditions i), ii), iii) is a weakly admissible vector equilibrium problem for $r-1$ measures with total masses $m_{j}=1-\frac{j}{r}, j=1, \ldots, r-1$. We set $\mu_{0}^{*}=\delta_{a}$, the Dirac point mass at $a>0$ and $m_{0}=1$. Also $\mu_{r}^{*}=0$ and $m_{r}=0$. Then by the discussion in Section 2, there is a unique minimizer $\left(\mu_{1}^{*}, \ldots, \mu_{r-1}^{*}\right)$ for the extended functional. We are going to construct the measures $\mu_{j}^{*}$ explicitly. We show that these measures have densities with respect to Lebesgue measures that decay as $|x|^{-1-1 / r}$ as $|x| \rightarrow \infty$, and that for $j=1, \ldots, r-1$,

$$
2 U^{\mu_{j}^{*}}=U^{\mu_{j-1}^{*}}+U^{\mu_{j+1}^{*}} \quad \text { on } \Delta_{j} .
$$

This implies that also also

$$
2 \tilde{U}^{\mu_{j}^{*}}=\tilde{U}^{\mu_{j-1}^{*}}+\tilde{U}^{\mu_{j+1}^{*}} \quad \text { on } \Delta_{j} .
$$

Then by Lemma 2.1 it follows that $\left(\mu_{1}^{*}, \ldots, \mu_{r-1}^{*}\right)$ is the minimizer for the extended functional, and since the function $x \mapsto \log \left(1+|x|^{2}\right)$ is integrable with respect to each of the measures, it then also follows that it is the minimizer of (3.1). We will see at the end of the proof that (3.2) and (3.3) hold as well.

We use a geometric construction based on the Riemann surface for the mapping $w=z^{1 / r}$. The Riemann surface has $r$ sheets

$$
\mathcal{R}_{j}= \begin{cases}\mathbb{C} \backslash \Delta_{1}=\mathbb{C} \backslash(-\infty, 0], & \text { for } j=1, \\ \mathbb{C} \backslash\left(\Delta_{j-1} \cup \Delta_{j}\right)=\mathbb{C} \backslash \mathbb{R}, & \text { for } j=2, \ldots, r-1, \\ \mathbb{C} \backslash \Delta_{r-1}, & \text { for } j=r,\end{cases}
$$

where $\mathcal{R}_{j}$ is connected to $\mathcal{R}_{j+1}$ along $\Delta_{j}$ for $j=1, \ldots, r-1$ in the usual crosswise manner. There is one point at infinity that connects all $r$ sheets. Note that $\mathcal{R}_{r}=\mathbb{C} \backslash(-\infty, 0]$ if $r$ is even, and $\mathcal{R}_{r}=\mathbb{C} \backslash[0, \infty)$ if $r$ is odd.

The Riemann surface has genus zero and $z=w^{r}$ is a rational parametrization of it. The rational function

$$
\Psi(w)=\frac{1}{r w^{r-1}\left(w-a^{1 / r}\right)}, \quad z=w^{r},
$$

is meromorphic on the Riemann surface with a simple pole at $z=a$, a pole of order $r-1$ at $z=0$, and a zero of order $r$ at $z=\infty$. We use $\Psi_{j}$ to denote its restriction to $\mathcal{R}_{j}$. Explicitly, we then have

$$
\Psi_{1}(z)=\frac{1}{r z^{1-1 / r}\left(z^{1 / r}-a^{1 / r}\right)}, \quad z \in \mathcal{R}_{1}=\mathbb{C} \backslash(-\infty, 0]
$$

with the principal branch of the $r$ th roots. On the other sheets we have the same formula but with different choices of $r$ th roots in $z^{1-1 / r}$ and $z^{1 / r}$. We always use $a^{1 / r}>0$.

The cuts $\Delta_{j}$ are oriented from left to right, and we use $\Psi_{j, \pm}(s), s \in \Delta_{j}$, to denote the limit of $\Psi_{j}(z)$ as $z \rightarrow s$ with $\pm \operatorname{Im} z>0$. Then we define for $j=1, \ldots, r-1$,

$$
d \mu_{j}^{*}(s)=\frac{1}{2 \pi i}\left(\Psi_{j,+}(s)-\Psi_{j,-}(s)\right) d s, \quad s \in \Delta_{j} .
$$

This defines a real measure on $\Delta_{j}$ since $\Psi_{j,-}(s)=\overline{\Psi_{j,+}(s)}$ for $s \in \Delta_{j}$, but a priori it could be a signed measure. Suppose the density vanishes at an interior point $s \in \Delta_{j}$. Then $\Psi_{j, \pm}(s)$ is real, which implies that $\Psi(w)$ is real for some $w \in \mathbb{C}$ with $w^{r}=s$. From the formula (3.4) for $\Psi$ it then easily follows that $w$ is real. However, if $w$ would be real and positive then $w^{r}$ would be on $[0, \infty)$ on the first sheet, and if $w$ would be real and negative then $w^{r}$ would be on $\mathbb{R} \backslash \Delta_{r-1}$ 
on the $r$ th sheet. Since $w^{r}=s$ is on one of the cuts, we have a contradiction and we see that the density (3.6) does not vanish at an interior, and therefore has a constant sign.

We compute the total masses by contour integration as in [9]. We consider $j=1$ first. Then by $(3.6)$

$$
\int d \mu_{j}^{*}(s)=\frac{1}{2 \pi i} \int_{C} \Psi_{1}(s) d s
$$

where $C$ is a contour that starts at $-\infty$ and follows the upper side of the cut $\Delta_{1}=(-\infty, 0]$, and goes back to $-\infty$ on the lower side of the cut. We deform the contour to a big circle $|s|=R$, and we pick up a residue condition from the pole at $s=a$. From (3.5) we calculate the residue as

$$
\lim _{z \rightarrow a}(z-a) \Psi_{1}(z)=\frac{1}{r a^{1-1 / r}} \lim _{z \rightarrow a} \frac{z-a}{z^{1 / r}-a^{1 / r}}=1 .
$$

Hence

$$
\int d \mu_{1}^{*}(s)=1-\lim _{R \rightarrow \infty} \frac{1}{2 \pi i} \int_{|s|=R} \Psi_{1}(s) d s
$$

with the circle $|s|=R$ oriented counterclockwise. Since for every $j=1, \ldots, r-1$,

$$
\Psi_{j}(z)=\frac{1}{r z}+O\left(z^{-1-1 / r}\right) \quad \text { as } z \rightarrow \infty,
$$

which easily follows from (3.4), we find

$$
\int d \mu_{1}^{*}(s)=1-\frac{1}{r}
$$

Now consider $2 \leq j \leq r-1$. Then by (3.6) and the fact that $\Psi_{j-1, \pm}=\Psi_{j, \mp}$ on $\Delta_{j-1}$,

$$
\begin{aligned}
\int d \mu_{j-1}^{*}(s)-\int d \mu_{j}^{*}(s)= & \frac{1}{2 \pi i} \int_{\Delta_{j-1}}\left(-\Psi_{j,-}(s)-\Psi_{j,+}(s)\right) d s \\
& -\frac{1}{2 \pi i} \int_{\Delta_{j}}\left(\Psi_{j,+}(s)-\Psi_{j,-}(s)\right) d s \\
= & \frac{1}{2 \pi i} \int_{\mathbb{R}}\left(\Psi_{j,-}(s)-\Psi_{j,+}(s)\right) d s,
\end{aligned}
$$

since $\Delta_{j-1} \cup \Delta_{j}=\mathbb{R}$. Again by contour deformation this is

$$
\int d \mu_{j-1}^{*}(s)-\int d \mu_{j}^{*}(s)=\lim _{R \rightarrow \infty} \frac{1}{2 \pi i} \int_{|s|=R} \Psi_{j}(s) d s=\frac{1}{r},
$$

where we used (3.7). Together with (3.8) we conclude that

$$
\int d \mu_{j}^{*}(s)=1-\frac{j}{r}, \quad j=1, \ldots, r-1 .
$$

Since the total masses are positive, and the densities of the measures do not change sign, it now also follows that the measures are positive.

We introduce the Cauchy transforms of the measures

$$
F_{j}(z)=\int \frac{d \mu_{j}^{*}(s)}{z-s}, \quad z \in \mathbb{C} \backslash \Delta_{j} .
$$


Then by a similar contour integration argument, where now we pick up a residue contribution at $s=z$, while there is no contribution from infinity, we get

$$
F_{1}(z)=\frac{1}{2 \pi i} \int_{C} \frac{\Psi_{1}(s)}{z-s} d s=\frac{1}{z-a}-\Psi_{1}(z), \quad z \in \mathbb{C} \backslash(-\infty, 0]
$$

and for $j=2, \ldots, r-1$,

$$
F_{j-1}(z)-F_{j}(z)=\frac{1}{2 \pi i} \int_{\mathbb{R}} \frac{\Psi_{j,-}(s)-\Psi_{j,+}(s)}{z-s} d s=\Psi_{j}(z), \quad z \in \mathbb{C} \backslash \mathbb{R},
$$

where $F_{r}(z)=0$. The identity $\Psi_{j,+}=\Psi_{j+1,-}$ on $\Delta_{j}$ then leads to

$$
F_{j,+}(x)+F_{j,-}(x)=F_{j-1}(x)+F_{j+1}(x), \quad x \in \Delta_{j},
$$

for $j=2, \ldots, r-1$. By (3.9) and (3.10), the identity (3.11) also holds for $j=1$, if we agree that

$$
F_{0}(z)=\frac{1}{z-a} .
$$

The measures have a density that decays like $|s|^{-1-1 / r}$ as $|s| \rightarrow \infty$. This easily follows from the definitions (3.4) and (3.6). Then $s \mapsto \log \left(1+s^{2}\right)$ is integrable for these measures, and the usual logarithmic potentials exist. By Sokhotskii-Plemelj formulas we have

$$
2 \frac{d}{d x} U^{\mu_{j}^{*}}(x)=F_{j,+}(x)+F_{j,-}(x), \quad x \in \Delta_{j} .
$$

Clearly also

$$
\frac{d}{d x} U^{\mu_{j \pm 1}^{*}}(x)=F_{j \pm 1}(x), \quad x \in \Delta_{j}
$$

Then by integrating (3.11) we obtain

$$
2 U^{\mu_{j}^{*}}(x)=U^{\mu_{j-1}^{*}}(x)+U^{\mu_{j+1}^{*}}(x), \quad x \in \Delta_{j} .
$$

There is no constant of integration in (3.12) since

$$
U^{\mu_{i}^{*}}(x)=\left(1-\frac{i}{r}\right) \log |x|+o(1) \quad \text { as }|x| \rightarrow \infty,
$$

for each $i \in\{j-1, j, j+1\}$ and $\Delta_{j}$ is unbounded.

Thus we have reached the identity (3.12) that we aimed for, as discussed in the beginning of the proof. It remains to verify (3.2) and (3.3).

By (3.12) we obtain for $j=1, \ldots, r-1$,

$$
I\left(\mu_{j}^{*}\right)=\int U^{\mu_{j}^{*}} d \mu_{j}^{*}=\frac{1}{2} \int\left(U^{\mu_{j-1}^{*}}+U^{\mu_{j+1}^{*}}\right) d \mu_{j}^{*}=\frac{1}{2}\left(I\left(\mu_{j-1}^{*}, \mu_{j}^{*}\right)+I\left(\mu_{j}^{*}, \mu_{j+1}^{*}\right)\right),
$$

which implies

$$
\sum_{j=1}^{r-1} I\left(\mu_{j}^{*}\right)=\sum_{j=1}^{r-2} I\left(\mu_{j}^{*}, \mu_{j+1}^{*}\right)+\frac{1}{2}\left(I\left(\mu_{0}^{*}, \mu_{1}^{*}\right)+I\left(\mu_{r-1}^{*}, \mu_{r}^{*}\right)\right) .
$$

We recall that $\mu_{0}^{*}=\delta_{a}$ and $\mu_{r}^{*}=0$ and we obtain

$$
\sum_{j=1}^{r-1} I\left(\mu_{j}^{*}\right)-\sum_{j=1}^{r-2} I\left(\mu_{j}^{*}, \mu_{j+1}^{*}\right)=\frac{1}{2} I\left(\delta_{a}, \mu_{1}^{*}\right)=\frac{1}{2} \int \log \frac{1}{|x-a|} d \mu_{1}^{*}(x),
$$

which is the identity in (3.2). 
Finally, we recall that by (3.9) and (3.5)

$$
F_{1}(z)=\frac{1}{z-a}-\frac{1}{r z^{1-1 / r}\left(z^{1 / r}-a^{1 / r}\right)}, \quad z \in \mathbb{C} \backslash(-\infty, 0],
$$

which after integration leads to

$$
\int \log (z-s) d \mu_{1}^{*}(s)=\log (z-a)-\log \left(z^{1 / r}-a^{1 / r}\right) .
$$

The constant of integration vanishes since both sides behave like $(1-1 / r) \log z+o(1)$ as $z \rightarrow \infty$. Taking real parts we find (3.3).

We next extend Proposition 3.1 from point masses $\delta_{a}$ with $a>0$ to general measures with compact support on $(0, \infty)$.

Proposition 3.2. Let $r \geq 2$ be an integer. Let $\mu$ be a probability measure on $(0, \infty)$ with compact support. Then there is a unique vector $\left(\mu_{1}^{*}, \ldots, \mu_{r-1}^{*}\right)$ of measures that minimizes the energy functional

$$
\sum_{j=1}^{r-1} I\left(\mu_{j}\right)-\sum_{j=1}^{r-2} I\left(\mu_{j}, \mu_{j+1}\right)-I\left(\mu, \mu_{1}\right)
$$

among all vectors of measures satisfying for every $j=1, \ldots, r-1$,

i) $\operatorname{supp}\left(\mu_{j}\right) \subset \Delta_{j}$,

ii) $\mu_{j}\left(\Delta_{j}\right)=1-\frac{j}{r}$, and

iii) $I\left(\mu_{j}\right)<+\infty$.

Moreover,

$$
\begin{aligned}
& \sum_{j=1}^{r-1} I\left(\mu_{j}^{*}\right)-\sum_{j=1}^{r-2} I\left(\mu_{j}^{*}, \mu_{j+1}^{*}\right)-I\left(\mu, \mu_{1}^{*}\right) \\
& \quad=-\frac{1}{2} I\left(\mu, \mu_{1}^{*}\right)=-\frac{1}{2} \iint \log \left|\frac{x^{1 / r}-y^{1 / r}}{x-y}\right| d \mu(x) d \mu(y) .
\end{aligned}
$$

Proof. For $\mu=\delta_{a}$ this was done in Proposition 3.1.

Let $\left(\mu_{1}^{*}(a), \ldots, \mu_{r-1}^{*}(a)\right)$ be the vector of measures that we obtain from $\delta_{a}$ as in Proposition 3.1. Then for a general probability measure $\mu$ on $(0, \infty)$ with compact support, we put

$$
\mu_{j}^{*}=\int \mu_{j}^{*}(a) d \mu(a), \quad j=1, \ldots, r-1 .
$$

These are well-defined positive measures satisfying i), ii) and iii) of the proposition. The measures $\mu_{j}^{*}(a)$ have a density that decays as $|x|^{-1-1 / r}$ as $|x| \rightarrow \infty$, and the same will be true for the measures $\mu_{j}^{*}$ since $\mu$ is compactly supported. Thus the logarithmic potentials exist, and

$$
U^{\mu_{j}^{*}}=\int U^{\mu_{j}^{*}(a)} d \mu(a)
$$

The identity

$$
2 U^{\mu_{j}^{*}(a)}=U^{\mu_{j-1}^{*}(a)}+U^{\mu_{j+1}^{*}(a)} \quad \text { on } \quad \Delta_{j}
$$


holds for every $a>0$ by Proposition 3.1. Integrating this with respect to $a$ and using Fubini's theorem, we obtain

$$
2 U^{\mu_{j}^{*}}=U^{\mu_{j-1}^{*}}+U^{\mu_{j+1}^{*}} \quad \text { on } \Delta_{j} .
$$

As in the proof of Proposition 3.1 this leads to the first identity of (3.13).

For $j=1$ we get

$$
U^{\mu_{1}^{*}}(z)=\int U^{\mu_{1}^{*}(a)}(z) d \mu(a)=\int \log \left|\frac{z^{1 / r}-a^{1 / r}}{z-a}\right| d \mu(a)
$$

see (3.3). Changing $z$ and $a$ to $x$ and $y$, and integrating over $d \mu(x)$, we obtain the second identity of $(3.13)$.

\section{Proofs of Theorems 1.1 and 1.2}

Theorem 1.1 is the special case $q=1$ of Theorem 1.2 and so it suffices to prove Theorem 1.2.

Proof. Let $\nu_{0}$ be a probability measure on $(0, \infty)$ with compact support, and let $\left(\nu_{-q+1}, \ldots, \nu_{-1}\right.$, $\left.\nu_{0}, \nu_{1}, \ldots, \nu_{r-1}\right)$ be the minimizing vector of measures for (1.9) under the assumptions i), ii) and iii) of Theorem 1.2, with $\nu_{0}$ fixed.

Then it is easy to see that $\left(\nu_{1}, \ldots, \nu_{r-1}\right)$ is the minimizer for the vector energy problem of Proposition 3.2 with $\mu=\nu_{0}$, and hence by (3.13),

$$
\sum_{j=1}^{r-1} I\left(\nu_{j}\right)-\sum_{j=1}^{r-2} I\left(\nu_{j}, \nu_{j+1}\right)-I\left(\nu, \nu_{1}\right)=-\frac{1}{2} \iint \log \left|\frac{x^{1 / r}-y^{1 / r}}{x-y}\right| d \nu_{0}(x) d \nu_{0}(y) .
$$

Similarly, $\left(\nu_{-1}, \ldots, \nu_{-q+1}\right)$ is the minimizer for the vector energy problem of Proposition 3.2 with $\mu=\nu_{0}$ and $q$ instead of $r$. Thus by (3.13) again,

$$
\sum_{j=-q+1}^{-1} I\left(\nu_{j}\right)-\sum_{j=-q+1}^{-2} I\left(\nu_{j}, \nu_{j+1}\right)-I\left(\nu_{-1}, \nu_{0}\right)=-\frac{1}{2} \iint \log \left|\frac{x^{1 / q}-y^{1 / q}}{x-y}\right| d \nu_{0}(x) d \nu_{0}(y) .
$$

The identity also holds in case $q=1$, since then $\nu_{-1}^{*}=0$ and both sides of (4.2) are 0 .

From (4.1) and (4.2) we find that for a fixed $\nu_{0}$, the minimum of the energy functional (1.9) taken over all $\nu_{j}$, for $j=-q+1,-1, j=1, \ldots, r-1$ satsifying items i), ii) of Theorem 1.2 , is equal to

$$
\begin{aligned}
J\left(\nu_{-q+1}, \ldots, \nu_{0}, \ldots, \nu_{r-1}\right)= & I\left(\nu_{0}\right)-\frac{1}{2} \iint \log \left|\frac{x^{1 / q}-y^{1 / q}}{x-y}\right| d \nu_{0}(x) d \nu_{0}(y) \\
& -\frac{1}{2} \iint \log \left|\frac{x^{1 / r}-y^{1 / r}}{x-y}\right| d \nu_{0}(x) d \nu_{0}(y)+\int \tilde{V}(x) d \nu_{0}(x),
\end{aligned}
$$

which reduces to (1.7) with $\nu_{0}$ instead of $\nu$. Thus the component $\nu_{0}^{*}$ of the minimizer for the vector energy (1.6) is also the minimizer of (1.7) over probability measures on $[0, \infty)$. of

We finally prove that $\nu_{0}^{*}$ has compact support. Let $\rho=\nu_{-1}^{*}+\nu_{1}^{*}$. Then $\nu_{0}^{*}$ is the minimizer

$$
\tilde{I}(\nu)-\tilde{I}(\nu, \rho)+\int \tilde{V} d \nu
$$

among probability measures $\nu$ on $[0, \infty)$. 
If $x \mapsto \log \left(1+|x|^{2}\right)$ would be integrable with respect to $\nu_{0}^{*}$ then it would also be the minimizer of

$$
\begin{aligned}
I(\nu) & -\tilde{I}(\nu, \rho)+\int\left(\tilde{V}(x)+\log \left(1+|x|^{2}\right)\right) d \nu(x) \\
& =I(\nu)+\int\left(\widehat{V}(x)-\int \log \frac{\sqrt{1+s^{2}}}{x-s} d \rho(s)\right) d \nu
\end{aligned}
$$

which is a usual minimization problem for one measure with an external field

$$
\widehat{V}(x)+\int \log \frac{x-s}{\sqrt{1+s^{2}}} d \rho(s)
$$

that is continuous on $(0, \infty)$ (since $\widehat{V}$ is continuous and $\rho$ is a measure on $(-\infty, 0]$ ).

It is easy to see that $x-s>\sqrt{1+s^{2}}$ for $x>1$ and $s<0$. Thus $\int \log \frac{x-s}{\sqrt{1+s^{2}}} d \rho(s)>0$ for $x>1$, and it follows from (1.8) that

$$
\lim _{x \rightarrow \infty}\left(\widehat{V}(x)+\int \log \frac{x-s}{\sqrt{1+s^{2}}} d \rho(s)-\log \left(1+x^{2}\right)\right)=+\infty,
$$

which guarantees that (4.4) has a minimizer with compact support. This minimizer also minimizes (4.3) and thus coincides with $\nu_{0}^{*}$ which thus has compact support.

Theorem 1.2 is now fully proved.

\section{A final remark}

We consider the minimization problem for (1.2) with $\theta=1 / r$. From Theorem 1.1 we obtain the following result that gives conditions that guarantee that the Cauchy transform of the minimizing measure is an algebraic function.

Proposition 5.1. Let $\theta=1 / r$ be rational and suppose the external field $V:[0, \infty) \rightarrow \mathbb{R}$ is such that $V^{\prime}$ is a rational function. Let $\mu^{*}$ be the probability measure that minimizes (1.2) among all probability measures on $[0, \infty)$ and suppose that $\mu^{*}$ is supported on a finite union of intervals Then its Cauchy transform

$$
F(z)=\int \frac{d \mu^{*}(s)}{z-s}
$$

is the solution of an algebraic equation of degree $r+1$. That is, there exist rational functions $c_{j}(z)$ for $j=0, \ldots, r$, such that

$$
\sum_{j=0}^{r} c_{j}(z) F(z)^{j}+F(z)^{r+1}=0, \quad z \in \mathbb{C} .
$$

Proof. We turn to the vector equilibrium problem from Theorem 1.1 and denote the minimizing vector by $\left(\mu_{0}^{*}, \ldots, \mu_{r-1}^{*}\right)$ with $\mu_{0}^{*}=\mu^{*}$. The variational conditions are

$$
2 U^{\mu^{*}}(x)-U^{\mu_{1}^{*}}(x)+V(x) \begin{cases}=\ell, & \text { for } x \in \operatorname{supp}\left(\mu^{*}\right), \\ \geq \ell, & \text { for } x \in[0, \infty),\end{cases}
$$

and for $j=1, \ldots, r-1$,

$$
2 U^{\mu_{j}^{*}}(x)-U^{\mu_{j-1}^{*}}(x)-U^{\mu_{j+1}^{*}}(x)=0 \quad \text { for } \quad x \in \Delta_{j},
$$


where $\mu_{r}^{*}=0$. We write

$$
F_{j}(z)=\int \frac{d \mu_{j}^{*}(s)}{z-s}, \quad j=1, \ldots, r
$$

and we find by differentiating the variational conditions

$$
\begin{array}{ll}
F_{0,+}^{\prime}(x)+F_{0,-}^{\prime}(x)=F_{1}(x)+V^{\prime}(x), & x \in \operatorname{supp}\left(\mu^{*}\right), \\
F_{j,+}^{\prime}(x)+F_{j,-}^{\prime}(x)=F_{j-1}(x)+F_{j+1}(x), & x \in \Delta_{j},
\end{array}
$$

for $j=1, \ldots, r-1$.

We construct a Riemann surface $\mathcal{R}$ with $r+1$ sheets $\mathcal{R}_{j}, j=0, \ldots, r$ given by

$$
\begin{aligned}
& \mathcal{R}_{0}=\mathbb{C} \backslash \operatorname{supp}\left(\mu^{*}\right), \\
& \mathcal{R}_{1}=\mathbb{C} \backslash\left((-\infty, 0] \cup \operatorname{supp}\left(\mu^{*}\right)\right), \\
& \mathcal{R}_{j}=\mathbb{C} \backslash\left(\Delta_{j-1} \cup \Delta_{j}\right), \quad \text { for } j=2, \ldots, r-1, \\
& \mathcal{R}_{r}=\mathbb{C} \backslash \Delta_{r-1},
\end{aligned}
$$

where $\mathcal{R}_{0}$ is connected to $\mathcal{R}_{1}$ along $\operatorname{supp}\left(\mu^{*}\right)$ and $\mathcal{R}_{j}$ is connected to $\mathcal{R}_{j+1}$ along $\Delta_{j}$ for $j=$ $1, \ldots, r-1$ in the usual crosswise manner. After adding points at infinity we obtain a compact Riemann surface, $\operatorname{since} \operatorname{supp}\left(\mu^{*}\right)$ consists of a finite union of intervals.

We define a function $\Psi$ on $\mathcal{R}$ by specifying on each of the sheets

$$
\Psi(z)= \begin{cases}V^{\prime}(z)-F_{0}(z), & \text { for } z \in \mathcal{R}_{0}, \\ F_{j-1}(z)-F_{j}(z), & \text { for } z \in \mathcal{R}_{j}, \quad j=1, \ldots, r .\end{cases}
$$

Then $\Psi$ is meromorphic on each of sheets (since $V^{\prime}$ is a rational function). Moreover, the variational conditions (5.1) tell us that $\Psi$ extends to a meromorphic function on the full Riemann surface $\mathcal{R}$. Then also $V^{\prime}-\Psi$ is a meromorphic function on $\mathcal{R}$ which agrees with $F_{0}$ on the zero sheet. Therefore $F=F_{0}$ satisfies an algebraic equation of degree $r+1$.

Note that $\mu^{*}$ minimizes $I(\mu)+\int\left(V-U^{\mu_{1}^{*}}\right) d \mu$ among all probability measures $\mu$ on $[0, \infty)$, and the external field $V-U^{\mu_{1}^{*}}$ is real analytic on $(0, \infty)$. If it were also real analytic at 0 , then it would follow from results in [8] that $\mu^{*}$ is supported on a finite union of intervals. Maybe the methods of [8] can be adapted to the present situation, and then the assumption in Proposition 5.1 about the finite number of intervals would be unnecessary.

The Riemann surface in the proof of Proposition 5.1 has genus 0 if and only if $\operatorname{supp}\left(\mu^{*}\right)=$ $[0, a]$ for some $a>0$. This is the case if $V(x)=x$, and for more general conditions see [7, Theorem 1.8]. We note that this Riemann surface also appears in the paper of Forrester, Liu and Zinn-Justin [12], see Fig. 1 in that paper.

\section{Acknowledgements}

The author is supported by long term structural funding-Methusalem grant of the Flemish Government, by KU Leuven Research Grant OT/12/073, by the Belgian Interuniversity Attraction Pole P07/18, and by FWO Flanders projects G.0934.13 and G.0864.16.

\section{References}

[1] Aptekarev A.I., Kuijlaars A.B.J., Hermite-Padé approximations and ensembles of multiple orthogonal polynomials, Russ. Math. Surv. 66 (2011), 1133-1199. 
[2] Beckermann B., Kalyagin V., Matos A.C., Wielonsky F., Equilibrium problems for vector potentials with semidefinite interaction matrices and constrained masses, Constr. Approx. 37 (2013), 101-134, arXiv:1105.3088.

[3] Bloom T., Levenberg N., Totik V., Wielonsky F., Modified logarithmic potential theory and applications, Int. Math. Res. Not., to appear, arXiv:1502.06925.

[4] Borodin A., Biorthogonal ensembles, Nuclear Phys. B 536 (1999), 704-732, math.CA/9804027.

[5] Butez R., Large deviations principle for biorthogonal ensembles and variational formulation for the DykemaHaagerup distribution, arXiv:1602.07201.

[6] Cheliotis D., Triangular random matrices and biorthogonal ensembles, arXiv:1404.4730.

[7] Claeys T., Romano S., Biorthogonal ensembles with two-particle interactions, Nonlinearity 27 (2014), 24192444, arXiv:1312.2892.

[8] Deift P., Kriecherbauer T., McLaughlin K.T.-R., New results on the equilibrium measure for logarithmic potentials in the presence of an external field, J. Approx. Theory 95 (1998), 388-475.

[9] Duits M., Kuijlaars A.B.J., An equilibrium problem for the limiting eigenvalue distribution of banded Toeplitz matrices, SIAM J. Matrix Anal. Appl. 30 (2008), 173-196, arXiv:0704.0378.

[10] Eichelsbacher P., Sommerauer J., Stolz M., Large deviations for disordered bosons and multiple orthogonal polynomial ensembles, J. Math. Phys. 52 (2011), 073510, 16 pages, arXiv:1102.0792.

[11] Forrester P.J., Liu D.-Z., Raney distributions and random matrix theory, J. Stat. Phys. 158 (2015), 10511082, arXiv:1404.5759.

[12] Forrester P.J., Liu D.-Z., Zinn-Justin P., Equilibrium problems for Raney densities, Nonlinearity 28 (2015), 2265-2277, arXiv:1411.4091.

[13] Forrester P.J., Wang D., Muttalib-Borodin ensembles in random matrix theory - realisations and correlation functions, arXiv:1502.07147.

[14] Hardy A., Kuijlaars A.B.J., Weakly admissible vector equilibrium problems, J. Approx. Theory 164 (2012), 854-868, arXiv:1110.6800.

[15] Kuijlaars A.B.J., Multiple orthogonal polynomial ensembles, in Recent Trends in Orthogonal Polynomials and Approximation Theory, Contemp. Math., Vol. 507, Amer. Math. Soc., Providence, RI, 2010, 155-176, arXiv:0902.1058.

[16] Kuijlaars A.B.J., Stivigny D., Singular values of products of random matrices and polynomial ensembles, Random Matrices Theory Appl. 3 (2014), 1450011, 22 pages, arXiv:1404.5802.

[17] Muttalib K.A., Random matrix models with additional interactions, J. Phys. A: Math. Gen. 28 (1995), L159-L164, cond-mat/9405084.

[18] Neuschel T., Van Assche W., Asymptotic zero distribution of Jacobi-Piñeiro and multiple Laguerre polynomials, J. Approx. Theory 205 (2016), 114-132, arXiv:1509.04542.

[19] Nikishin E.M., Sorokin V.N., Rational approximations and orthogonality, Translations of Mathematical Monographs, Vol. 92, Amer. Math. Soc., Providence, RI, 1991.

[20] Saff E.B., Totik V., Logarithmic potentials with external fields, Grundlehren der Mathematischen Wissenschaften, Vol. 316, Springer-Verlag, Berlin, 1997. 